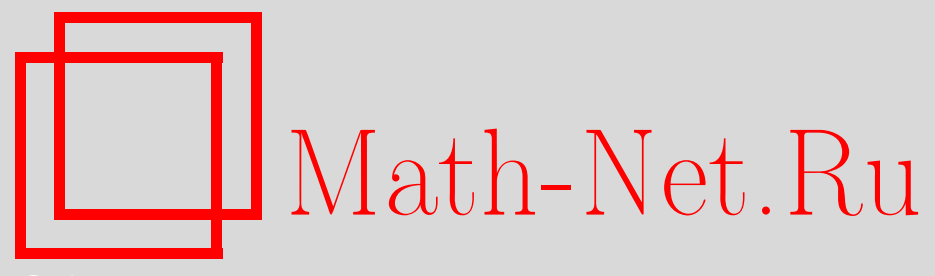

Е. Бакаев, Прыжки в правильном многоугольнике, Квант, 2020, номер 3, 34-38

DOI: https://doi.org/10.4213/kvant20200304

Использование Общероссийского математического портала Math-Net.Ru подразумевает, что вы прочитали и согласны с пользовательским соглашением http://www.mathnet.ru/rus/agreement

Параметры загрузки:

IP : 54.198 .64 .247

26 апреля 2023 г., 14:22:08 


\section{ПрыЖкИ в \\ правильном \\ МНОГОУгОЛЬНИКе}

\section{E. БAKAEB}

ЭТОЙ СТАТЬЕ МЫ РАССМОТРИМ

несколько решений задачи, предлагавшейся в одном из недавних туров Конкурса имени А.П.Савина. А именно, задачи 20:

В каждой вершине правильного 4k-угольника сидело по блохе. Каждая блоха дружит с двумя своими соседями. Половина блох - красные, половина - синие, причем красные и синие блохи чередуются. Блохи начали прыгать. В первую секунду прыгнули красные: каждая прыгнула в точку, симметричную ей относительно прямой, соединяющей двух ее друзей. Во вторую секунду аналогичным образом прыгнули все синие блохи. В третью секунду снова прыгнули все красные и т.д. Докажите, что через $k$ секунд все блохи снова окажутся в вершинах некоторого правильного $4 k$-угольника.
PUC. 1

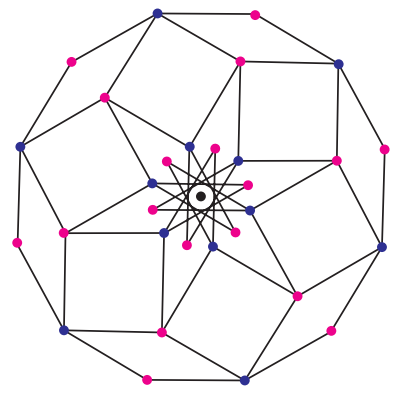

На рисунке 1 приведен пример того, как красные блохи, сидящие в вершинах правильного 12-угольника, сделают три прыжка.

\section{Первый способ}

Пусть точка $O$ - центр исходного многоугольника. Рассмотрим двух соседних блох в точках $A$ и $B$. Они все время будут находиться на прямых $O A$ и $O B$ соответственно. Рассмотрим треугольник $A O B$ и выясним, как движутся эти две блохи - очевидно, что другие пары соседних

DOI: https://doi.org/10.4213/kvant20200304 блох будут скакать по своим треугольникам аналогичным образом.

C каждым прыжком внутри угла $A O B$ будет откладываться отрезок, равный $A B$. Выясним, какими будут углы возникающих равнобедренных треугольников. Пусть на $i$-м прыжке блоха прыгает в точку $P_{i}$, при этом образуется равнобедренный треугольник $\Delta_{i}$ с углами $\alpha_{i}$ при основании. Первые два треугольника изображены на рисунке 2. Из

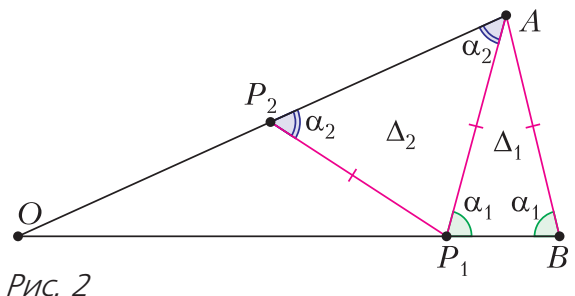

того что треугольник $A O B$ равнобедренный и $\angle A O B=\frac{360^{\circ}}{4 k}=\frac{90^{\circ}}{k}$, несложно найти

$$
\alpha_{1}=90^{\circ}-\frac{45^{\circ}}{k}, \alpha_{2}=90^{\circ}-3 \cdot \frac{45^{\circ}}{k} .
$$

Мы хотим получить явную формулу для $\alpha_{i}$, т.е. выразить этот угол через $i$. Естественно делать это по индукции, но мы поступим немного иначе. Рассмотрев последовательные треугольники $\Delta_{i-1}, \quad \Delta_{i}, \quad \Delta_{i+1}$,

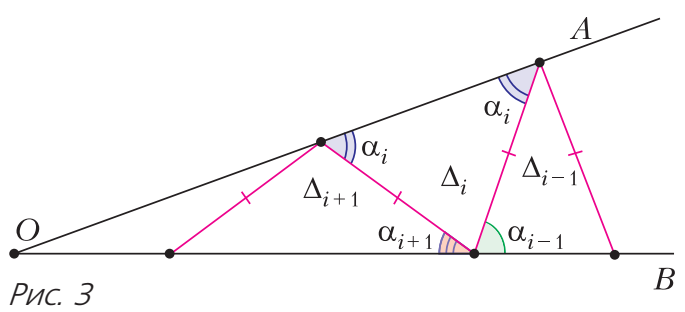

можно заметить, что $2 \alpha_{i}=\alpha_{i-1}+\alpha_{i+1}$ (рис.3). Если преобразовать это равенство в $\alpha_{i}-\alpha_{i-1}=\alpha_{i+1}-\alpha_{i}$, то станет видно, что последовательность $\alpha_{i}$ является арифметической прогрессией. Зная ее начало, мы можем записать формулу общего члена:

$$
\alpha_{i}=90^{\circ}-(2 i-1) \cdot \frac{45^{\circ}}{k} .
$$

Подумаем теперь, где кончится эта последовательность треугольников. Очередной треугольник $\Delta_{i}$ Удастся построить внутри 
угла $A O B$, если угол $\alpha_{i}=90^{\circ}-(2 i-1) \cdot \frac{45^{\circ}}{k}$ не больше $\angle A O B=\frac{90^{\circ}}{k}$, т.е. при $i \leq k-1$.

Итак, треугольник $\Delta_{k}$ - это первый треугольник из рассматриваемой последовательности треугольников, который не поместится целиком внутри угла $A O B$ (рис.4). Так

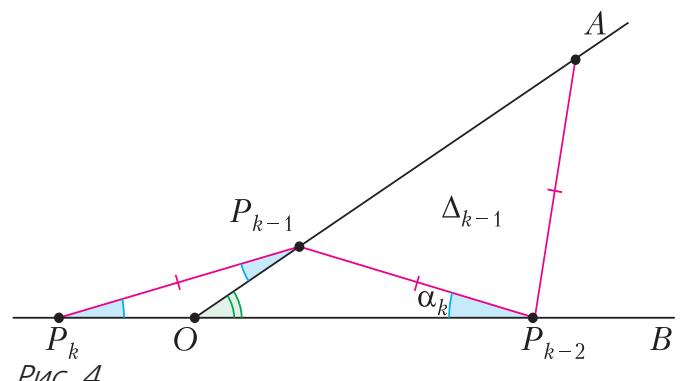

Рис. 4

как $\alpha_{k}=\frac{45^{\circ}}{k}=\frac{\angle A O B}{2}$, то треугольник $O P_{k} P_{k-1}$ равнобедренный и $O P_{k}=O P_{k-1}$.

Получается, что после $k$-го прыжка эти две блохи, а значит и все блохи, будут снова равноудалены от точки О. Пара блох, которые изначально сидели в противоположных вершинах $4 k$-угольника, либо обе останутся на тех же лучах, исходящих из $O$, либо займут лучи друг друга. Поэтому снова все блохи будут сидеть в вершинах правильного $4 k$-угольника. Задача решена.

Эта конструкция - прыжки фиксированной длины с одной стороны угла на другую рассматривалась в задаче Н.Б.Васильева, вошедшей в «Задачник «Кванта» под номером M190:

На плоскости дань две пересекающиеся прямые $а$ и $b$. . прямой а на расстоянии меньше 1 от прямой b, сидит блоха. Затем блоха последовательно прыгает в точки $B_{1}$, $A_{2}, \quad B_{2}, A_{3}, B_{3}, \ldots$, руководствуясь следуюшими правилами. Во-первых, точки $A_{1}, A_{2}$, $A_{3}, \ldots$ лежат на прямой $а$, точки $B_{1}, B_{2}, B_{3}, \ldots$ - на прямой $b$. Bo-smopыx, $\quad 1=A_{1} B_{1}=B_{1} A_{2}=$ $=A_{2} B_{2}=B_{2} A_{3}=A_{3} B_{3}=\ldots \quad B$-mpemьих, наконеч, точка $A_{n}$ не совпадает с $A_{n+1}$, кроме случая $A_{n} B_{n} \perp$ а (аналогично, $B_{n}$ совпадает c $B_{n+1}$, только если $\left.B_{n} A_{n+1} \perp b\right)$. Нетрудно видеть, ито этими тремя условиями последовательность прыжков определена одно- значно. Докажите, что если угол между прямыми а и b измеряется рачиональным числом градусов, то путь блохи будет периодическим, т.е. в некоторый момент она попадет в начальную точку $A_{1}$ и затем будет последовательно проходить те же самые точки $B_{1}, A_{2}, B_{2}, A_{3}, B_{3}, \ldots$, как в начале пути; а если угол измеряется иррациональным числом градусов, то блоха не попадет ни в какую точку более двух раз.

Видно, что в конкурсной задаче 20 рассматривается частный случай конструкции из задачи M190 - когда угол между прямыми равен $\frac{90^{\circ}}{k}$ для натуральных $k$.

\section{Второй способ}

Соединим каждую блоху со следующей по часовой стрелке. Будем следить за тем, как этот набор из $n$ векторов меняется при прыжках $(n=4 k)$. Пусть на некотором шаге одна из блох прыгает из точки $B$ в точку $B^{\prime}$, a ее

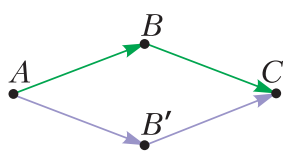

Pис. 5 друзья сидят в точках $A$ и $C$ (рис.5). Тогда $A B C B^{\prime}$ - ромб, и в рассматриваемом наборе векторы $\overrightarrow{A B}$ и $\overrightarrow{B C}$ заменятся на $\overrightarrow{A B^{\prime}}=\overrightarrow{B C}$ и $\overrightarrow{B^{\prime} C}=\overrightarrow{A B}$, т.е. поменя-

ются местами.

Таким образом, при каждом прыжке все векторы разбиваются на пары соседних и два вектора каждой пары меняются местами. Раз происходят только перестановки начального набора векторов, то для краткости записи можно рассматривать перестановку их индексов. Например, при $k=3$ последовательность перестановок будет такой, как в таблице (для наглядности четные и нечетные индексы покрашены в разные цвета):

\begin{tabular}{|rcccccccccccc|}
\hline Сначала & 1 & 2 & 3 & 4 & 5 & 6 & 7 & 8 & 9 & 10 & 11 & 12 \\
после 1-го прыжка & 2 & 1 & 4 & 3 & 6 & 5 & 8 & 7 & 10 & 9 & 12 & 11 \\
после 2-го прыжка & 11 & 4 & 1 & 6 & 3 & 8 & 5 & 10 & 7 & 12 & 9 & 2 \\
после 3-го прыжка & 4 & 11 & 6 & 1 & 8 & 3 & 10 & 5 & 12 & 7 & 2 & 9 \\
\hline
\end{tabular}

Заметим, что каждый раз четные индексы сдвигаются на одну позицию относительно нечетных. При этом и четные и нечетные индексы не меняют своего внутреннего порядка. Поэтому через $k$ прыжков получится такая последовательность: 


$$
1(2+2 k) 3(4+2 k) 5(6+2 k) \ldots
$$

Векторы $\overrightarrow{a_{i}}$ и $\overrightarrow{a_{i+2 k}}$ являются противоположными, поскольку изначально они шли вдоль противоположных сторон правильного 4k-угольника. Значит, последовательность векторов, которая получится через $k$ прыжков, можно записать так: $\overrightarrow{a_{1}},-\overrightarrow{a_{2}}, \overrightarrow{a_{3}},-\overrightarrow{a_{4}}, \ldots$ $\ldots, \overrightarrow{a_{n-1}},-\overrightarrow{a_{n}}$. Покажем теперь, что если двигаться по таким векторам в этом порядке, то мы будем совершать обход вершин правильного $n$-угольника.

Угол между соседними векторами $\overrightarrow{a_{i}}$ и $\overrightarrow{a_{i+1}}-$ это внешний угол правильного $4 k$-угольника, он равен $\frac{360^{\circ}}{4 k}$. Такой же угол образуют диагонали $A_{1} A_{2 k}$ и $A_{2 k} A_{4 k-1}$ правильного $4 k$ угольника $A_{1} A_{2} \ldots A_{4 k}$ (рис.6). Это показывает, что совершается обход вершин правильного $4 k$-угольника по его «почти самым длинным» диагоналям длины, равной стороне исходного $4 k$-угольника (рис.7).

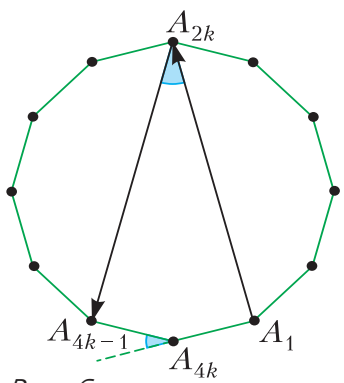

Pис. 6

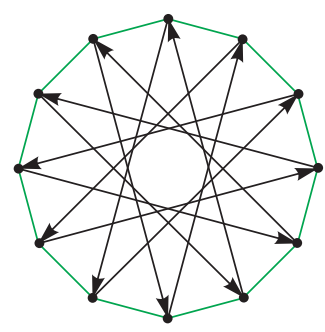

Pис. 7

\section{Третий способ}

Вернемся к ломаной с равными звеньями, вершины которой лежат на сторонах угла $A O B$ - как в первом решении (рис.8). Рассмотрим два соседних звена $P_{k-1} P_{k}$ и $P_{k} P_{k+1}-$ они образуют равнобедренный треугольник. Отразим $\angle A O B$ относительно той стороны, на которой лежит $P_{k}$; пусть точке $P_{k+1}$ симметрична точка $P_{k+1}^{\prime}$. Тогда точки $O, P_{k-1}, P_{k}$ и $P_{k+1}^{\prime}$ лежат на одной окружности (это следует из того, что два противоположных угла в сумме дают $180^{\circ}$ ).

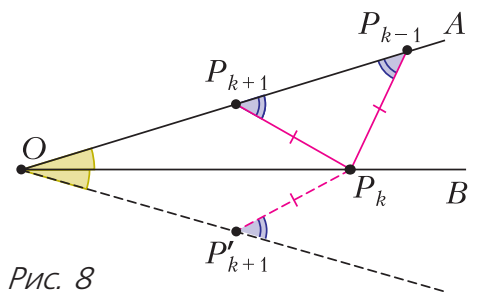

Эта конструкция встречается часто. Для треугольников $O P_{k} P_{k-1}$ и $O P_{k} P_{k+1}$ выполняется так называемый четвертый признак «равенства» треугольников: «по двум сторонам и углу не между ними». Такие треугольники не обязательно равны (для этого вывода нужны дополнительные условия), но если они не равны, то, в частности, из них можно сложить вписанный четырехугольник.

Возможен также другой случай взаимного расположения точек: $P_{k+1}$ может попасть не на луч $O A$, а на его дополнение (рис.9). Но и тогда те же четыре точки лежат на одной окружности.

Эти два случая охватывают все возможные

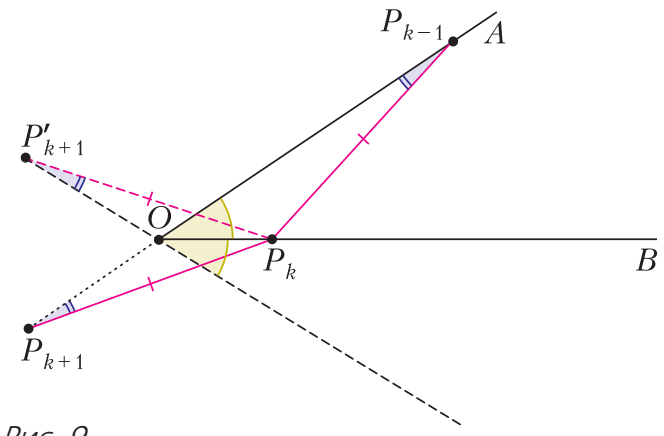

Pnc. 9

варианты расположения точек, помимо вырожденных (т.е. когда некоторые из этих 4 точек совпадают), но для них факт продолжает оставаться верным.

Итак, мы разобрались, что происходит при таком отражении одного звена ломаной. Теперь повторим это действие много раз. Если так и дальше отражать углы, то получится пучок лучей с равными углами между соседними лучами. А ломаная «распрямится» в такую ломаную, у которой каждая следующая вершина лежит на следующем луче (рис.10). И раз любые три соседние

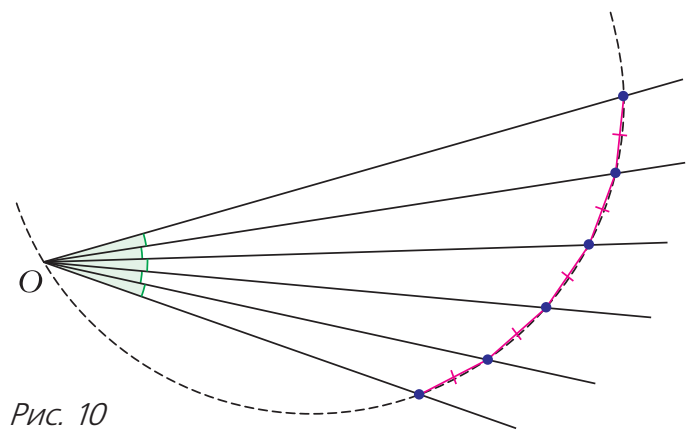


точки лежат на одной окружности с точкой $O$, то и все они лежат на одной окружности.

Вернемся к исходной задаче о правильном многоугольнике. Первое звено ломаной - это его сторона $A B$. Тогда после «выпрямления» ломаной все точки будут лежать на окружности, описанной около $A O B$, где $O$ - центр правильного многоугольника (на рисунке 11 показан пример для $k=3$ ). Угол $A O B$

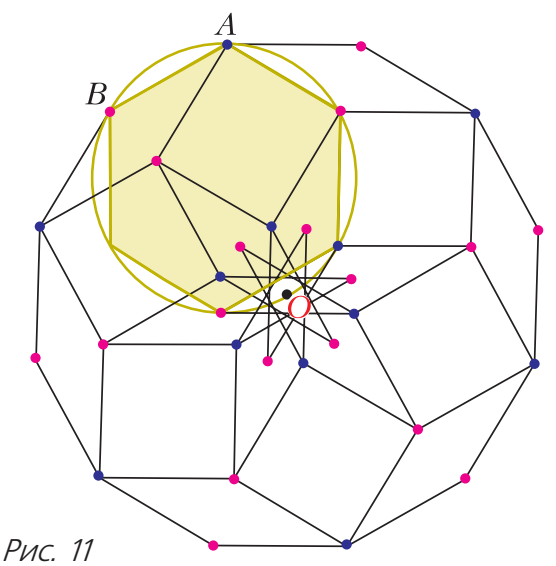

является вписанным в эту окружность и центральным углом описанной окружности правильного 4k-угольника, значит, градусные меры дуг $A B$ этих двух окружностей отличаются в 2 раза. Отсюда следует, что в описанную около $A O B$ окружность вписывается правильный $2 k$-угольник со стороной $A B$. Значит, он и является ломаной, в которую «выпрямится» исходная! Отсюда ясно, что места, на которые блохи прыгнули после $(k-1)$-го прыжка и после $k$-го прыжка, будут равноудалены от точки $O$ - ведь они, как и весь правильный $2 k$-угольник, симметричны относительно серединного перпендикуляра к стороне $A B$. Задача решена.

Зададимся еще парой естественных вопросов, возникающих при размышлении над исходной задачей.

Мы выяснили, как будут расположены блохи через $k$ прыжков. А как будут двигаться блохи после этого? Предлагаем подумать об этом самостоятельно.

Следующий вопрос такой. В задаче рассматривался $4 k$-угольник. Понятно, почему требуется, чтобы количество вершин было четным - чтобы правило, по которому прыгают блохи, было корректным. А что будет,

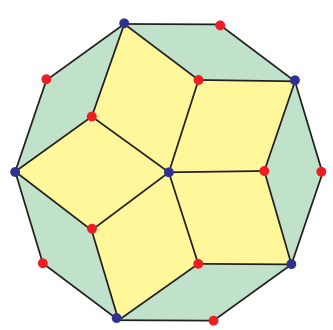

Pnc. 12

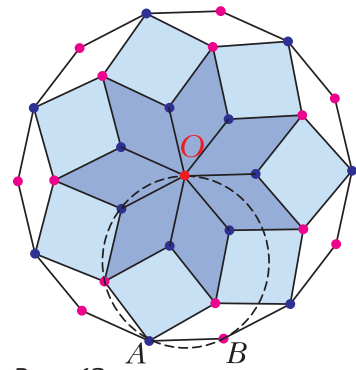

Pис. 13 если оно четное, но не делится на 4? Иными словами, что интересного можно заметить про аналогичную последовательность прыжков для $(4 k+2)$-угольника? На рисунках 12 и 13 приведены иллюстрации для $k=2$ и $k=3$ (иллюстрацию для $k=1$ предлагаем представить в уме).

Видно, что возникает разбиение правильного многоугольника на ромбы. После $k$-го прыжка половина блох попадает в центр - не только в рассмотренных случаях, а для всех $k$, и это можно доказать, используя рассмотренные нами подходы.

Если рассмотреть последовательность равнобедренных треугольников, как в первом способе, то получится, что $k$ таких треугольников целиком замощают треугольник $A O B$. Это, конечно, требует доказательства, но оно аналогично доказательству для $4 k$-угольника (и даже несколько проще его), и мы не будем приводить его здесь.

Отметим, что получившаяся конструкция любопытна сама по себе. Например, для $k=2$ она позволяет найти значение косинуса $36^{\circ}$ просто с помощью подобия треугольников $A B C$ и $A O B$ (рис.14):

$$
\left\{\begin{array}{l}
\frac{1}{x}=\frac{1+x}{1}, \\
1+x=2 \cos 36^{\circ} .
\end{array}\right.
$$

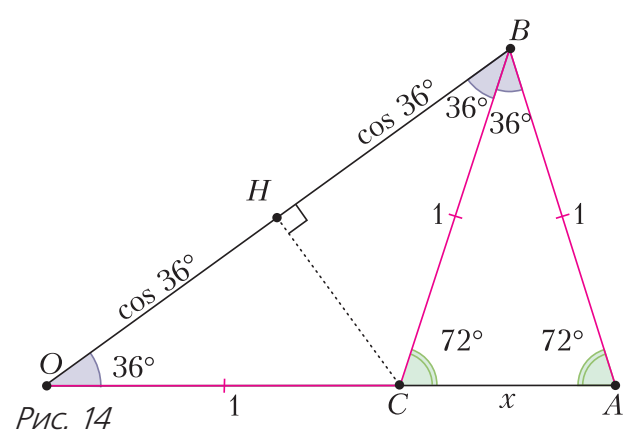


Также эта конструкция скрыта, например, в такой задаче: требуется найти угол, отмеченный знаком вопроса на рисунке 15. Подумайте, какое значение $k$ здесь лучше выбрать.

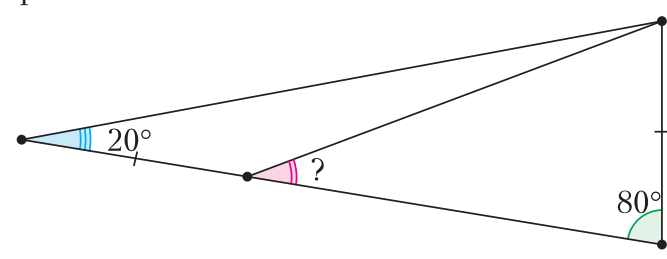

Pис. 15

Предлагаем самостоятельно подумать над тем, как применить к задаче про $(4 k+2)$-угольник идеи второго способа.

Третий способ дает нам такое короткое объяснение: число $\frac{4 k+2}{2}=2 k+1$ нечетно, и у правильного $(2 k+1)$-угольника со стороной $A B$ одна из вершин попадает в точку $O$ (см.рис.13).
Этот же подход из третьего способа можно применить и к задаче М190. Пусть вместо того, чтобы прыгать по сторонам одного угла, блоха перепрыгивает на следующий луч этого пучка (см. рис.10). Тогда получается, что она скачет по окружности с равными интервалами. Когда величина угла между исходными прямыми (т.е. $\angle A O B$ в наших обозначениях) выражается рациональным числом градусов, то и длина прыжка блохи по окружности тоже выражается рациональным числом градусов, значит, в какой-то момент ее путь зациклится. В ином случае длина прыжка блохи по окружности составляет иррациональное число градусов и она все время будет попадать в новые точки, т.е. расстояние от блохи до точки $O$ не может повториться три раза.

Статья Н.Б.Васильева «Последовательность прыжков» в «Кванте» № 11 за 1973 год посвящена другому подходу к задаче М190. Рекомендуем прочитать эту статью.

\section{Н А М П И ШІ У Т}

\section{Как выглядит график синуса?}

П.ПАНОВ

Необычный график функции $y=\sin (314 x)$ я обнаружил в одной из студенческих работ по электротехнике, откуда его и позаимствовал (рис.1). Конечно, так график синуса не может выглядеть ни в коем случае, и мы хотим разобраться в причинах этой аномалии, понять, в чем тут дело.

Прежде поясним, откуда в электротехнике берется функция $y=\sin (314 x)$. На самом деле она, как чертик, выскакивает из каждой розетки. Всем известно, что в России частота сетевого напряжения составляет $f=50$ Гц. После перехода к угловой частоте получаем $\omega=2 \pi f=100 \pi$ рад/с и после естественного

DOI: https://doi.org/10.4213/kvant20200305

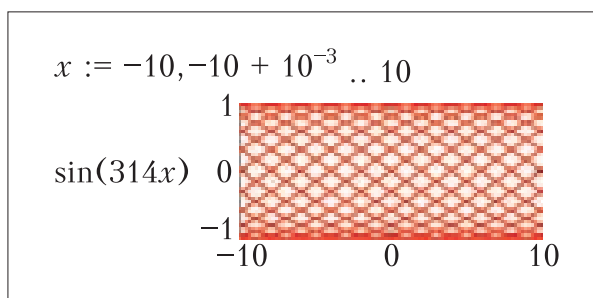

Рис. 1. "График» функции $y=\sin (314 x)$ составлен из точек вида $\left(x_{i} ; \sin \left(314 x_{i}\right)\right)$, где $x_{i}=i \cdot 10^{-3}$

округления имеем $\omega=314$ рад/с. При этом зависимость напряжения от времени принимает вид $U(t)=220 \sin (314 t) \mathrm{B}$. Отбросив постоянный множитель и заменив $t$ на $x$, получим ту самую функцию $y=\sin (314 x)$.

Отметим, что рисунок 1 был построен в среде Mathcad. При этом предваряющая его строка означает, что функция $y=\sin (314 x)$ вычисляется в точках $x_{i}, x_{i}=i \cdot 10^{-3}$, где $i$ пробегает все целые от $-10^{4}$ до $10^{4}$, и сама картинка состоит из точек с координатами

$$
\left(x_{i} ; \sin \left(314 x_{i}\right)\right) \text {. }
$$

